\title{
ChIP-seq Profiling Identifies Histone Deacetylase 2 Targeting Genes Involved in Immune and Inflammatory Regulation Induced by Calcitonin Gene-Related Peptide in Microglial Cells
}

\author{
Xingjing Guo, ${ }^{1,2}$ Dan Chen, ${ }^{3}$ Shuhong An $\mathbb{D}^{1},{ }^{1}$ and Zhaojin Wang $\mathbb{C}^{1}$ \\ ${ }^{1}$ Department of Human Anatomy, Shandong First Medical University \& Shandong Academy of Medical Sciences, Taian, China \\ ${ }^{2}$ Department of Physiology, Shandong First Medical University \& Shandong Academy of Medical Sciences, Taian, China \\ ${ }^{3}$ Experimental Center, Shandong University of Traditional Chinese Medicine, Jinan, China
}

Correspondence should be addressed to Shuhong An; shhan7766@126.com and Zhaojin Wang; zjwang@sdfmu.edu.cn

Received 16 April 2020; Accepted 7 July 2020; Published 4 August 2020

Guest Editor: Fabiano Carvalho

Copyright (c) 2020 Xingjing Guo et al. This is an open access article distributed under the Creative Commons Attribution License, which permits unrestricted use, distribution, and reproduction in any medium, provided the original work is properly cited.

Calcitonin gene-related peptide (CGRP) is a mediator of microglial activation at the transcriptional level. The involvement of the epigenetic mechanism in this process is largely undefined. Histone deacetylase (HDAC) $1 / 2$ are considered important epigenetic regulators of gene expression in activated microglia. In this study, we examined the effect of CGRP on HDAC2mediated gene transcription in microglial cells through the chromatin immunoprecipitation sequencing (ChIP-seq) method. Immunofluorescence analysis showed that mouse microglial cells (BV2) expressed CGRP receptor components. Treatment of microglia with CGRP increased HDAC2 protein expression. ChIP-seq data indicated that CGRP remarkably altered promoter enrichments of HDAC2 in microglial cells. We identified 1271 gene promoters, whose HDAC2 enrichments are significantly altered in microglia after CGRP treatment, including 1181 upregulating genes and 90 downregulating genes. Bioinformatics analyses showed that HDAC2-enriched genes were mainly associated with immune- and inflammation-related pathways, such as nitric oxide synthase (NOS) biosynthetic process, retinoic acid-inducible gene- (RIG-) like receptor signaling pathway, and nuclear factor kappa B (NF- $\kappa \mathrm{B})$ signaling pathway. The expression of these key pathways (NOS, RIG-I, and NF- $\kappa \mathrm{B}$ ) were further verified by Western blot. Taken together, our findings suggest that genes with differential HDAC2 enrichments induced by CGRP function in diverse cellular pathways and many are involved in immune and inflammatory responses.

\section{Introduction}

Microglia are innate immune cells of the central nervous system that are responsible for the excessive and chronic neuroinflammatory response following damage and disease [1, 2]. Calcitonin gene-related peptide (CGRP) as a mediator of glial cell activation plays a role as signaling molecule, mediating interactions between damaged neurons and surrounding glial cells [3]. Previous research has demonstrated that the presence of CGRP induced the activation of microglia at the transcriptional level through expression of the immediate-early genes in cultured microglia $[4,5]$.

CGRP operates via its receptor that, in many cell types, leads to a range of biological effects including those associated with neurons and microglia $[6,7]$. A functional CGRP receptor consist of 3 components, the calcitonin receptorlike receptor (CRLR), receptor activity-modifying protein 1 (RAMP1), and CGRP receptor component protein (CRCP) that couple the receptor to the cellular signal pathway leading to increased intracellular cAMP and activated protein kinase A (PKA) [8]. Accumulating evidence suggested that microglia express functional CGRP receptors, as treatment with CGRP triggers microglial activation by induction of c-fos gene expression, cAMP accumulation, and release of proinflammatory mediators in microglia $[4,5$, 9]. A previous study indicated that CGRP may act as a paracrine mediator to induce activation of glial activation $[10,11]$. Administration of CGRP in rat models of temporomandibular joint disorder contributed to the increased microglial marker of OX-42 expression and microglial 
activation in the spinal cord [12], suggesting a functional link between CGRP and microglial activation.

Histone deacetylase (HDAC) $1 / 2$ are essential for microglial survival and expansion during development [13]. Increased activity of HDAC2 is closely related to glial activation in a mouse model of retinal injury [14]. Selective inhibition of HDAC1 or 2 suppresses the expression of inflammatory cytokines in BV2 murine microglia activated with lipopolysaccharide (LPS) [15]. Previous data indicated that HDAC2 activates NF- $\kappa$ B and promotes NF- $\kappa$ B-dependent gene expression [16], which plays a crucial role in microglial activation [17].

The ability of CGRP to activate microglial cells at the level of transcription raises the question of whether the gene expression induced by CGRP associates with epigenetic regulation by HDAC2 in activated microglia. In the present study, we adopted chromatin immunoprecipitation followed by sequencing (ChIP-seq) to profile and compare the variation of HDAC2 enrichments in target genes at the genomewide level in the microglial cell line (BV2) from treatment of CGRP and control to gain a better understanding of a potential role for this peptide in the responses of microglia. In addition, the expression of CGRP receptor components was also explored in BV2 cells and Western blot was used to determine whether the expression of HDAC2 in microglia was influenced by CGRP, hoping that these studies could further understand the underlying regulatory mechanism of microglial activation by CGRP at the molecular level.

\section{Materials and Methods}

2.1. Cell Culture and Drug Administration. The mouse microglial cell line (BV2) was obtained from the Cell Bank of the Chinese Academy of Sciences (Beijing, China). Microglial cells were cultured in Dulbecco's modified Eagle's medium (DMEM, Gibco) supplemented with 10\% fetal bovine serum (FBS, Biological Industries) incubated at $37^{\circ} \mathrm{C}$ in an atmosphere of $5 \% \mathrm{CO}_{2}$. BV2 cells continuously stimulated with CGRP peptide $(1 \mu \mathrm{mol} / \mathrm{L}$, Tocris Bioscience, Cat. $\# 1161$ ) at 1,2, 4, and $6 \mathrm{~h}$, respectively. Cells without CGRP peptide were used as the control. To assess the possible impact of HDAC2 on the expression of iNOS, RIG-I, and $\mathrm{NF}-\kappa \mathrm{B}$ induced by CGRP, $0.5 \mathrm{mmol} / \mathrm{L}$ valproic acid (VPA, HDAC2 inhibitor, MedChemExpress, Cat. \#1069-66-5) were preapplied for $60 \mathrm{~min}$ and coapplied together with CGRP for $2 \mathrm{~h}$ at $37^{\circ} \mathrm{C}$.

2.2. Immunofluorescence of Cultured Cells. Immunofluorescence was performed essentially as described before [18]. BV2 cells were cultured on poly-L-lysine-coated coverslips in six-well plates. Following a single wash in phosphatebuffered saline (PBS), cultured microglial cells were fixed in $4 \%$ paraformaldehyde for $15 \mathrm{~min}$ at room temperature. Double-labeling immunofluorescence staining for primary antibodies against ionized calcium binding adaptor molecule 1 (Iba1, a marker for microglia; $1: 200$, Novus, Cat. \#NB 100-1028) and CRLR (1:200, Abcam, Cat. \#ab84467), RAMP1 ( $1: 200$, Sigma-Aldrich, Cat. \#SAB250086), or CRCP (1:200, Proteintech, Cat. \#14348-1-AP) on coverslip-cultured microglia was performed. Coverslips were incubated with a mixture of the two primary antibodies overnight. Following three washes with tris-buffered saline (TBS), coverslips were treated with a mixture of matching secondary antibodies (Jackson ImmunoResearch). The specificity of antibodies used was checked by Western blotting and/or omission of the primary antibodies. No specific immunoreactivity was detected in these controls.

2.3. Chromatin Immunoprecipitation. Chromatin was prepared from fixed mouse microglial cells (stimulated with $1 \mu \mathrm{mol} / \mathrm{L}$ CGRP, $2 \mathrm{~h}$ ) and sonicated fragments ranged in size from 200 to $1500 \mathrm{bp}$. Approximately $2 \times 10^{7}$ cell equivalents were used for each immunoprecipitation. ChIP was performed as described previously [19], using anti-HDAC2 antibody (Abcam, Cat. \#ab12169, ChIP Grade) or a control rabbit IgG.

2.4. Sequencing Library Preparation, Cluster Generation, and Sequencing. DNA samples were end-repaired, A-tailed, and adaptor-ligated using TruSeq Nano DNA Sample Prep Kit (Cat. \#FC-121-4002, Illumina), following the manufacturer's instructions. $\sim 200-1500 \mathrm{bp}$ fragments were size-selected using AMPure XP beads. The final size of the library was confirmed by Agilent 2100 Bioanalyzer. Samples were diluted to a final concentration of $8 \mathrm{pmol} / \mathrm{L}$, and cluster generation was performed on the Illumina cBot using HiSeq 3000/4000 PE Cluster Kit (Cat. \#PE-410-1001, Illumina), following the manufacturer's instructions. Sequencing was performed on Illumina HiSeq 4000 using HiSeq 3000/4000 SBS Kit (300 cycles) (Cat. \#FC-410-1003, Illumina), according to the manufacturer's instructions.

2.5. Data Collection and ChIP-seq Analysis. After sequencing platform-generated sequencing images, stages of image analysis and base calling were performed using Off-Line Basecaller software (OLB V1.8). Sequence quality was examined using the FastQC software. After passing Solexa CHASTITY quality filter, clean reads were aligned to mouse genome (UCSC MM10) using Bowtie software (V2.1.0) [20]. Aligned reads were used for peak calling of ChIP regions using MACS V1.4.2 [21]. Statistically significant ChIP-enriched regions (peaks) were identified by comparison of IP vs. input or comparison to a Poisson background model, using a $P$ value threshold of $10^{-4}$. Peaks in samples were annotated by the nearest gene using the newest UCSC RefSeq database [22]. The annotation of the peaks which were located within $-2 \mathrm{~kb}$ to $+2 \mathrm{~kb}$ around the corresponding gene TSS in samples can be found from the peaks-promoter-annotation.

2.6. Bioinformatics Analysis. The Gene Ontology (GO) functional and Kyoto Encyclopedia of Genes and Genomes (KEGG) pathway enrichment analyses were performed using the Database for Annotation, Visualization and Integrated Discovery (DAVID) and KEGG Orthology-Based Annotation System (KOBAS) online tools (http://www.geneontology.org and http://www.genome.jp/kegg) [23, 24]. 
2.7. Western Blotting. Western blot analysis was performed as described before [18]. Cultured microglial cells were lysed, and the protein was extracted. The protein lysate from each sample was separated electrophoretically on a $10 \%$ sodium dodecyl sulfate-polyacrylamide gel and then transferred to a polyvinylidene fluoride (PVDF) membrane. After blocking with $5 \%$ nonfat milk in TBS-T (containing $0.1 \%$ Tween20) for $2 \mathrm{~h}$, membranes were incubated with HDAC2 (Abcam, Cat. \#ab32117), NF- $\kappa$ B (Abcam, Cat. \#1559-1), RIG-I (Abcam, Cat. \#ab45428), and iNOS (Abcam, Cat. \#ab3523) in 5\% nonfat milk in TBS-T overnight at $4^{\circ} \mathrm{C}$. After washes with TBS-T, membranes were incubated with appropriate secondary antibodies for $2 \mathrm{~h}$. Results were visualized using an ECL chemiluminescence system. GAPDH rabbit $\mathrm{mAb}$ antibody (Cell Signaling Technology, Cat. \#2118) was also used as a probed control to ensure the loading of equivalent amounts of sample proteins. Band densities were compared in TotalLab software (version 2.01; Bio-Rad, Hercules, CA).

2.8. Statistical Analysis. Data are presented as the mean \pm standard error of the mean (SEM). For the analyses of Western blot data, Mann-Whitney $U$ tests were used for comparisons between two groups, and Kruskal-Wallis tests with Dunn's multiple comparisons post hoc tests were used for comparisons among multiple groups. The data from the Rotarod test were compared using Kruskal-Wallis tests with Dunn's multiple comparisons post hoc tests. Significance was defined by $P$ values $<0.05$.

\section{Results}

3.1. CGRP Increases HDAC2 Expression in Microglial Cells. To study the effect of CGRP on microglial cells, we first investigated the expression of CGRP receptor components on microglia. Figure 1(a) shows an example of colocalization of CRLR, RAMP1, and CRCP with the Ibal (microglial marker) immunoreactivity on microglial cells in culture. Nearly all of the Iba1-positive cells expressed CGRP receptor components CRLR, RAMP1, and CRCP.

The expression of HDAC2 protein in microglia was assessed by Western blot following treatment with CGRP for $0,1,2,4$, and $6 \mathrm{~h}$, respectively. As shown in Figure 1(b), CGRP treatment significantly increased the expression of the HDAC2 protein level in microglia $(P<0.05)$. CGRP was found to induce the expression of HDAC2 in a time-dependent manner with a maximal effect observed after $6 \mathrm{~h}$.

3.2. Genome-Wide Profile of HDAC2 Targets in Microglia after CGRP Treatment. To investigate the role of HDAC2 on microglia after treatment with CGRP, the profile of HDAC2 targets in microglial cells was analyzed using an Illumina HiSeq 4000 sequencing technique after stimulation with CGRP for $2 \mathrm{~h}$. Model-based Analysis of ChIP-seq (MACS, v1.4.2) software was used to detect the ChIPenriched regions (peaks) from ChIP-seq data. Differentially enriched regions with statistical significance between the CGRP-treated group and the control were identified by
Detecting Differential Chromatin Modification Sites from ChIP-seq Data with Biological Replicates (diffReps), cut-off: $\mathrm{FC}=2.0, P=10^{-4}$ ).

The ChIP-seq for the CGRP-treated microglial cells generated 21827 enriched regions (peaks), including 17646 up peaks and 4181 down peaks, compared with the control group. The peak distribution of ChIP-seq reads of HDAC2 was showed in Figures 2(a) and 2(b). We identified 1271 gene promoters, whose HDAC2 enrichments are significantly altered in microglial cells treated with CGRP, including 1181 upregulated genes and 90 downregulated genes (Table S1). The distribution of HDAC2-enriched promoters was mapped to proximal regions of transcription start sites (TSSs) of RefSeq genes (from about $-1800 \mathrm{bp}$ to $+1800 \mathrm{bp}$ of TSSs, Figure 2(c)).

3.3. GO Analysis of Peaks Relative to Annotated Genes. To further understand functions of annotated genes related to peaks, they were functionally classified using GO terminology. According to the functional annotation in GO database, key upregulated HDAC2 target genes were mostly enriched for biological process (BP) terms associated with positive regulation of $\mathrm{T}$ cell cytokine production (e.g., tumor necrosis factor receptor associated factor 6 (TRAF6), mucosaassociated lymphoid tissue lymphoma translocation gene 1 (MALT1)), histone acetylation (e.g., lysine acetyltransferase 8 (KAT8), KAT8 regulatory NSL complex subunit 2 (KANSL2)), activation of NF- $\kappa \mathrm{B}$-inducing kinase activity (e.g., ZFP91 zinc finger protein (ZFP91), TRAF6, and MALT1), and NOS biosynthetic process (e.g., Fc fragment of IgE receptor II (FCER2A), nucleotide binding oligomerization domain containing 2 (NOD2), prostaglandin E receptor 4 (PTGER4), and toll-like receptor 2 (TLR2)). When focusing on cellular components (CC), the most represented categories were BLOC-1 complex, nuclear exosome (RNase complex), and NADPH oxidase complex. The most represented categories for molecular function (MF) were related to $3^{\prime}, 5^{\prime}$-cGMP phosphodiesterase activity, histone acetyltransferase activity, nucleosomal DNA binding, and NADPH binding. Most genes are well-known immune and inflammatory responses (e.g., TRAF6, TLR2, and FCER2A). Association with immune- and inflammation-related genes seems therefore to be a feature of CGRP-mediated HDAC2 enrichments. GO enrichment terms of BP, CC, and MF for upregulated genes are shown in Figure 3(a).

Meanwhile, key dowregulated HDAC2 target genes were enriched in BP terms such as regulation of histone H3K9 acetylation, toll-like receptor 9 signaling pathway, astrocyte activation, and negative regulation of tumor necrosis factor signaling. The most represented categories for CC were related to SMN complex and nuclear lamina and MF terms such as cyclic nucleotide-gated ion channel activity and cGMP binding. GO enrichment terms of BP, $\mathrm{CC}$, and MF for downregulated genes are shown in Figure 3(b).

3.4. KEGG Pathway Analysis of Peaks Relative to Annotated Genes. KEGG pathway enrichment analysis was performed using the software KOBAS. The $P<0.05$ was set as the 

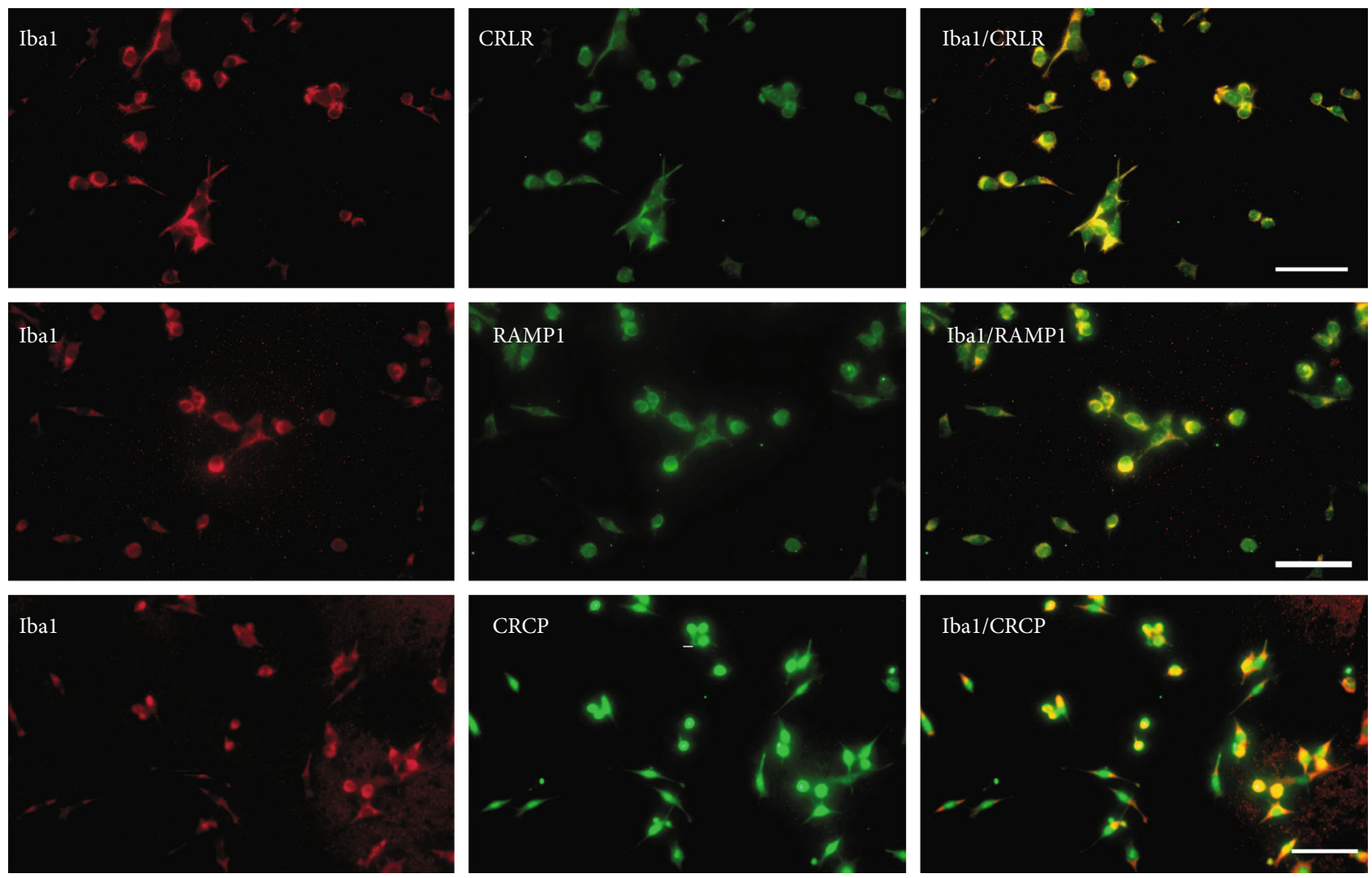

(a)
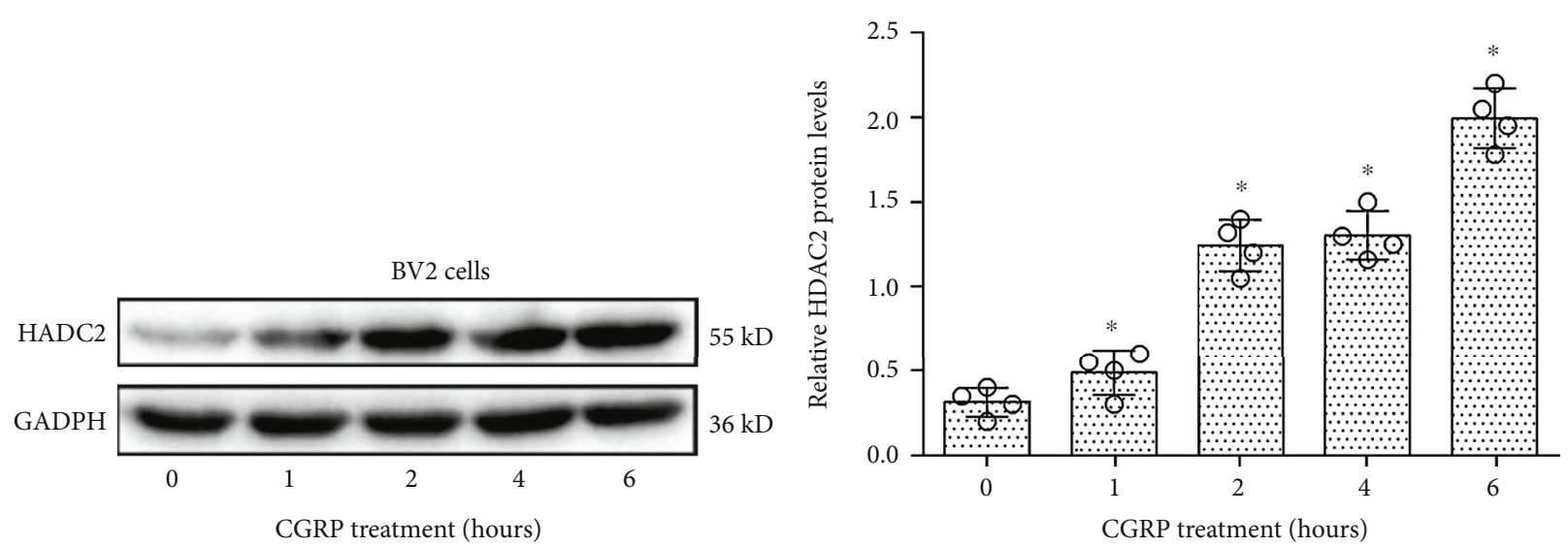

(b)

FIGURE 1: Cultured mouse microglial cells (BV2) expressed Iba1 and CGRP receptor components CRLR, RAMP1, or CRCP. (a) Expression of Ibal (a marker of microglia, red) and its colocalization with CRLR, RAMP1, or CRCP staining (green) in cultured microglial cells. Scale bar $40 \mu \mathrm{m}$. (b) Western blot analysis of HDAC2 expression in microglia with treatment with CGRP at $0,1,2,4$, and $6 \mathrm{~h}$, respectively. Relative amounts of proteins were calculated by normalizing to GAPDH. Data are presented as the mean \pm SEM $(N=4$ independent cell culture preparations, Mann-Whitney $U$ tests or Kruskal-Wallis tests). ${ }^{*} P<0.05$.

threshold of significant enrichment. Based on the KEGG pathway enrichment analysis, key upregulated genes were significantly enriched in 10 signaling pathways, such as the Fc epsilon RI signaling pathway, RIG-like receptor signaling pathway, NF- $\kappa \mathrm{B}$ signaling pathway, and T cell receptor signaling pathway, which were mostly related to immune and inflammatory responses (Figure 3(c)). From these data, KEGG enrichment analysis related to immune and inflammatory responses accounted for the enrichment score which also agree with the GO enrichment analysis above. However, none of downregulated genes was significantly enriched in any KEGG pathway.

3.5. Validation of Key Pathways from ChIP-seq by Western Blot. In order to explore whether or not CGRP involves the NOS biosynthetic process, RIG-like receptor signaling pathway, and NF- $\kappa \mathrm{B}$ signaling pathway, the expression of iNOS, RIG-I, and NF- $\kappa \mathrm{B}$ protein levels in microglial cells was 


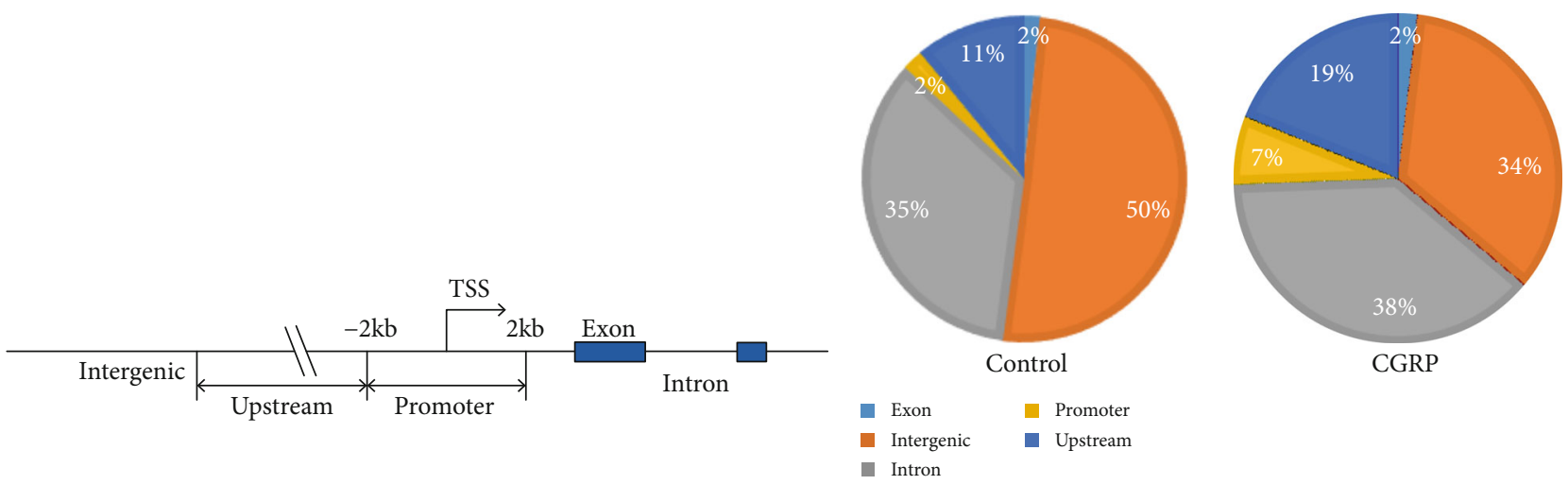

(a)

(b)

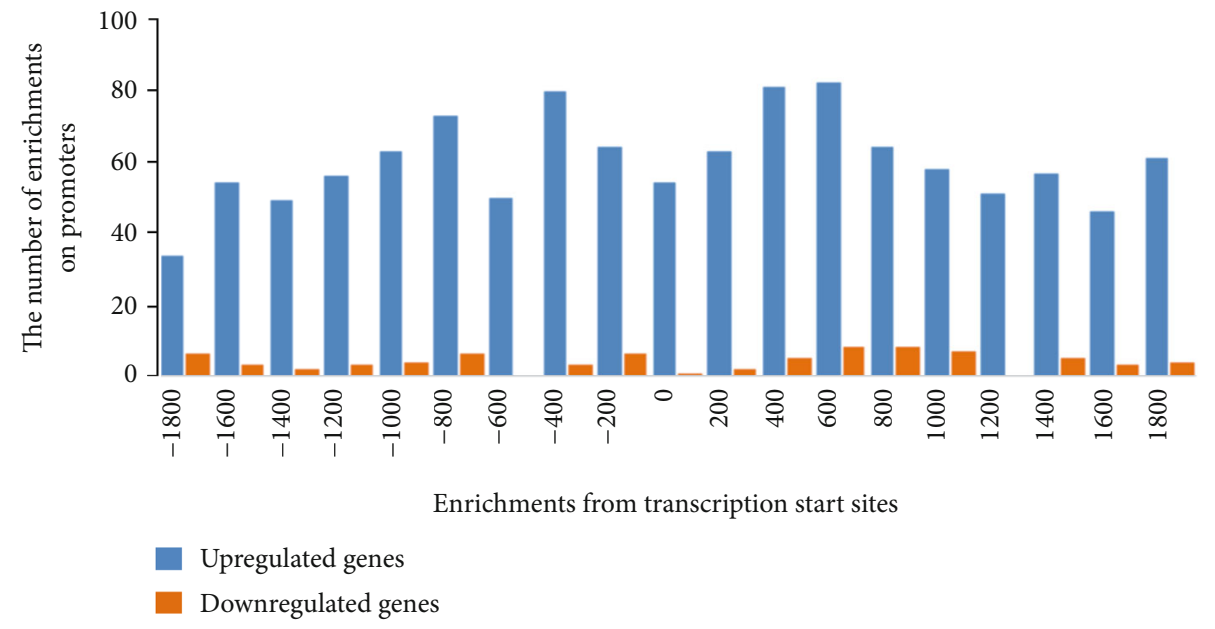

(c)

FIGURE 2: Effect of CGRP on the enriched region (peak) distribution of the ChIP-seq reads of HDAC2 in microglia treated with CGRP compared with the control. (a) Genome-wide distribution of enrichments relative to annotated genes. (b) The peak distribution of ChIPseq reads of HDAC2 in control and microglial cells treated with CGRP. (c) The distribution of HDAC2 peaks on promoters relative to gene transcription start sites (TSSs). Shown are HDAC2 peak frequencies relative to the distance from the nearest annotated TSS in microglial cells treated with CGRP.

assessed by Western blot after treatment with CGRP for 0,1 , 2, 4, and $6 \mathrm{~h}$, respectively. As shown in Figure 4(a), CGRP treatment significantly increased the expression of iNOS, RIG-I, and NF- $\kappa$ B protein levels in microglia $(P<0.05)$. CGRP was found to induce the expression of iNOS, RIG-I, and NF- $\kappa \mathrm{B}$ in a time-dependent manner with a maximal effect observed after $6 \mathrm{~h}$ which was well in agreement with the HDAC2 enrichment analysis observed by bioinformatics analysis. The results of Western blot analyses provided evidence that the ChIP-seq method for large-scale gene expression quantification was reliable.

3.6. HDAC2 Inhibitor Suppresses the Increase of iNOS, RIG-I, and NF- $\kappa B$ Expression Induced by CGRP. A recent report showed that HDAC2 increased the expression of iNOS in macrophages following inflammatory stimulation [25]. To test whether HDAC2 was involved in CGRPinduced expression of iNOS, RIG-I, and NF- $\kappa \mathrm{B}$ in this study, a HDAC2 inhibitor VPA was coapplied together with CGRP. As shown in Figure 4(b), CGRP increased the expression of iNOS, RIG-I, and NF- $\kappa$ B following treatment of microglia with CGRP, and the HDAC2 inhibitor VPA partially or completely blocked the CGRP-mediated upregulation of iNOS, RIG-I, and NF- $\kappa \mathrm{B}(P<0.05)$.

\section{Discussion}

Here, we mapped HDAC2 enrichment profiles induced by CGRP at these loci using ChIP-seq in mouse microglial cells and examined the influence of CGRP on HDAC2 enrichment profiles on promoter regions to test the hypothesis that the gene expression induced by CGRP was associated with HDAC2-mediated epigenetic regulation in microglia [15, 17]. ChIP-seq data showed that CGRP could substantially change HDAC2 enrichments on gene promoters, including 1181 upregulated genes and 90 downregulated genes, suggesting that CGRP could alter enrichment profiles of HDAC2 at promoters in a gene-specific manner.

HDAC2 is involved in regulating microglial activation and gene transcription $[14,15]$. Our results showed that 


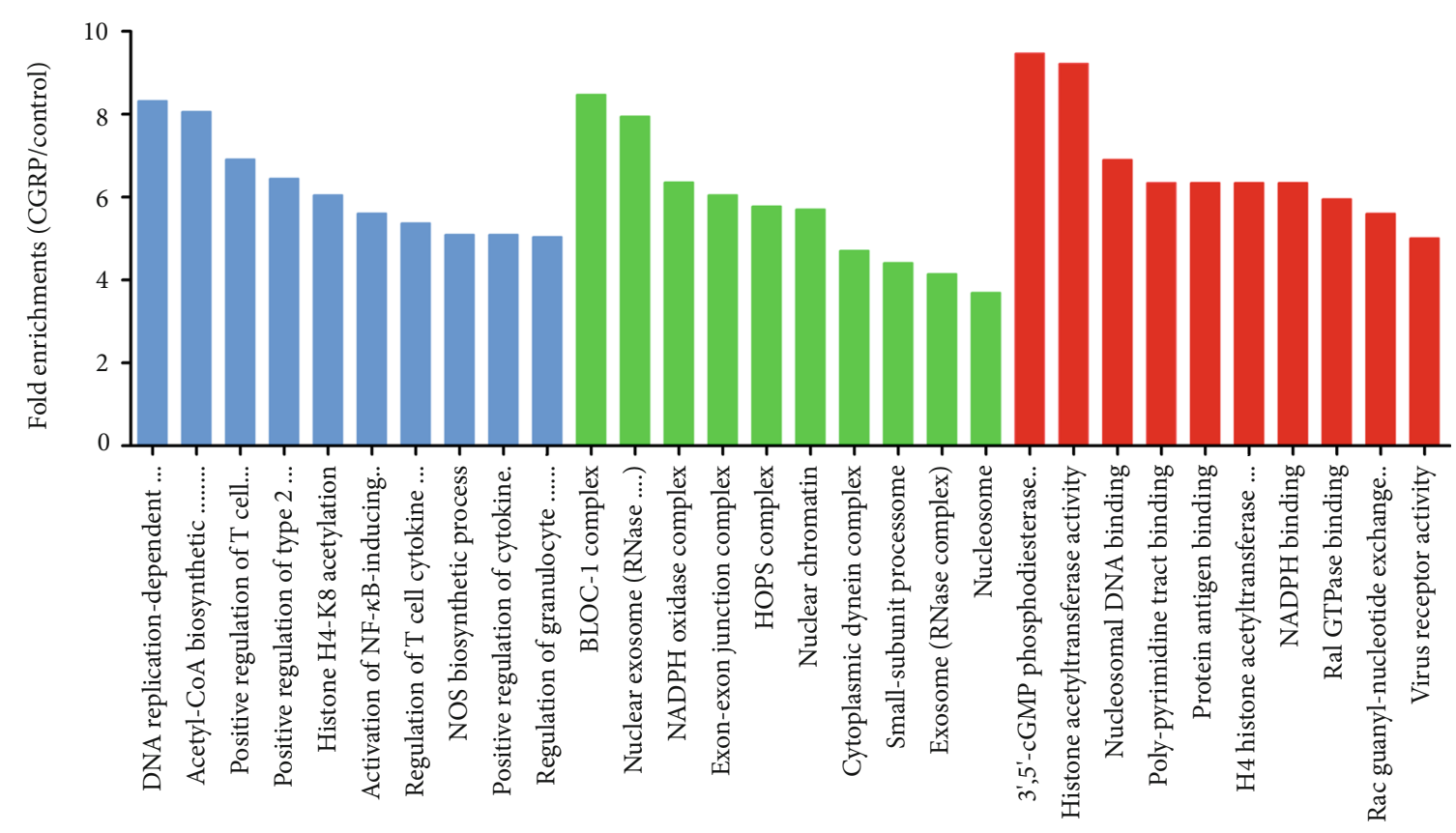

Biological process

Cellular component

Molecular function

(a)

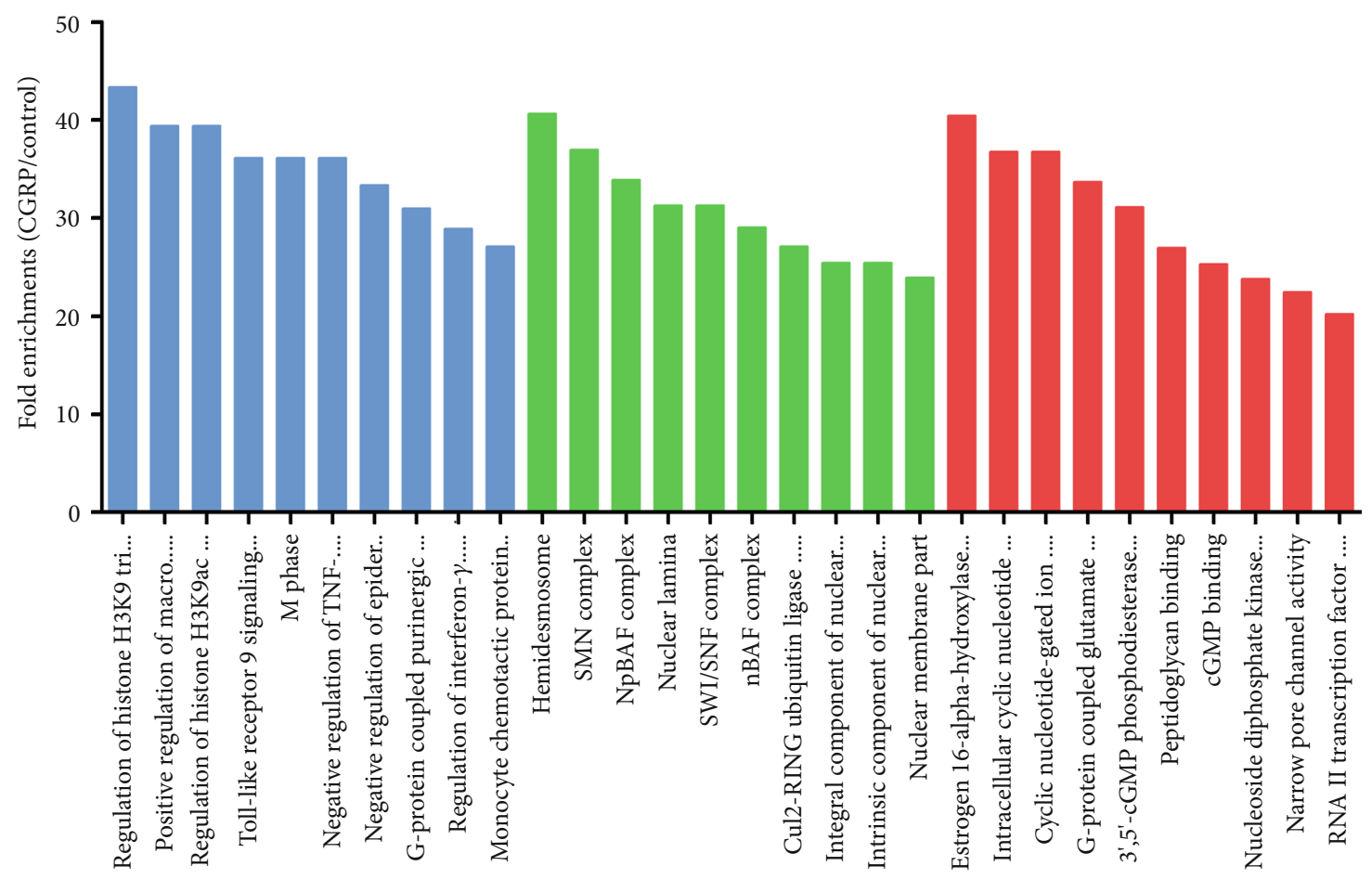

Biological process

Cellular component

Molecular function

(b)

FIgUre 3: Continued. 


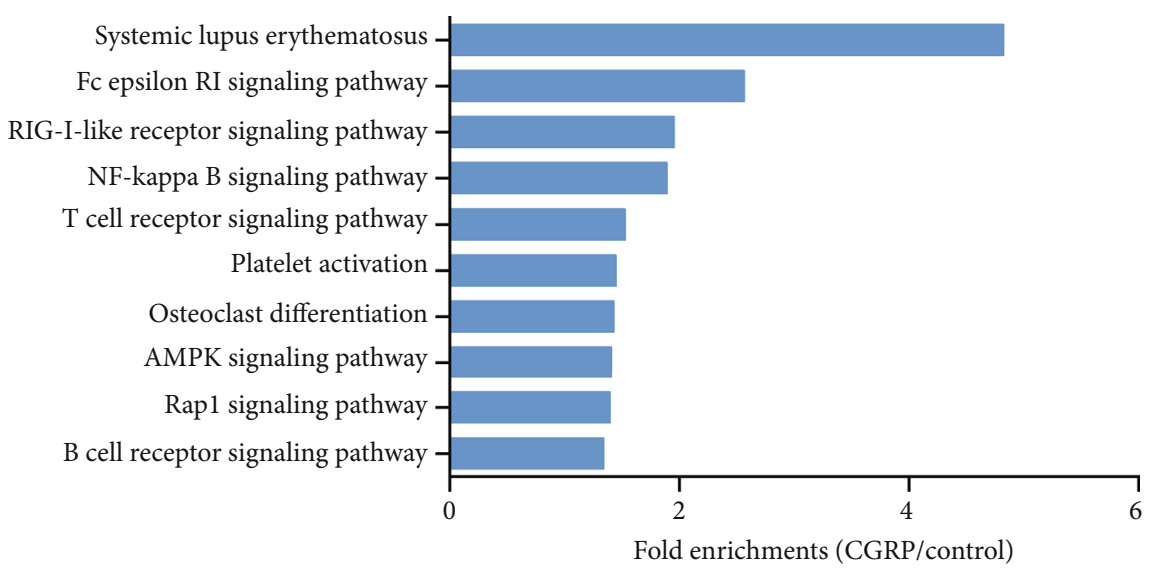

(c)

Figure 3: The Gene Ontology (GO) and Kyoto Encyclopedia of Genes and Genomes (KEGG) pathway analyses of genes with differentially enriched HDAC2 in microglial cells treated with CGRP. GO annotation of upregulated genes (a) or downregulated genes (b) with HDAC2 enrichments of the CGRP treatment group versus the control. Bar plots show the top ten fold enrichment values of the significant enrichment terms involving biological process (BP), cellular component (CC), and molecular function (MF). (c) KEGG pathway analysis of genes with differentially enriched HDAC2 in microglial cells treated with CGRP. The bar plot shows the top ten fold enrichment values of the significant enrichment pathway. Analysis by DAVID and KOBAS online tools (http://www.geneontology.org and http://www.genome.jp/ kegg).
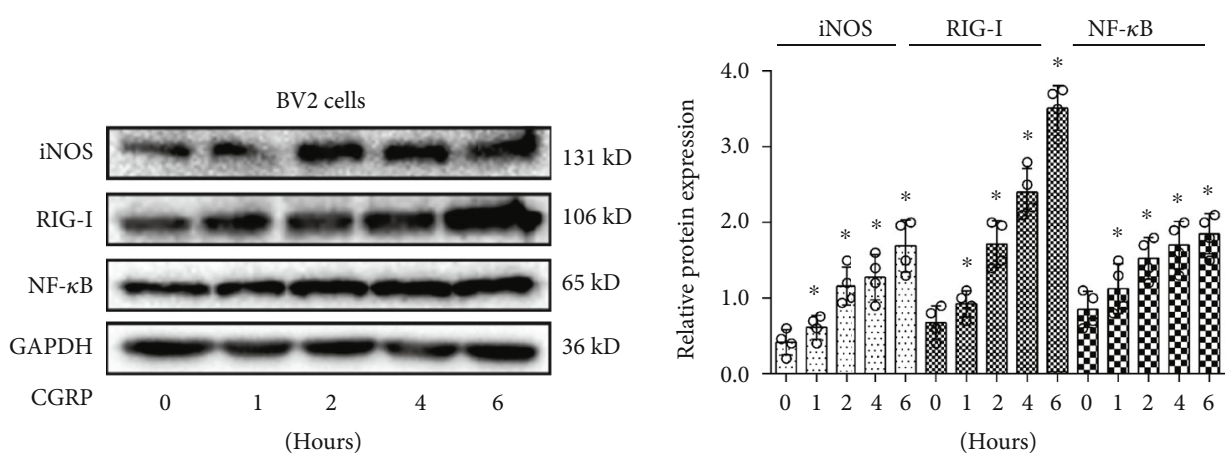

(a)
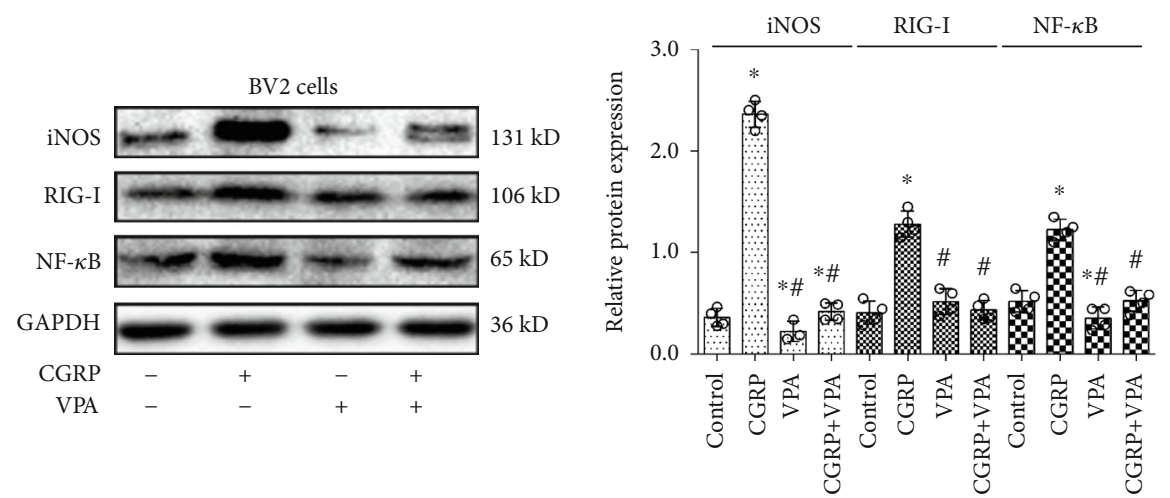

(b)

FIGURE 4: CGRP evokes increases in the expression of iNOS, RIG-I, and NF- $\kappa$ B in microglial cells in a time-dependent manner. (a) Western blot analysis of iNOS, RIG-I, and NF- $\kappa$ B expression in microglial cells with treatment of CGRP at $0,1,2,4$, and $6 \mathrm{~h}$, respectively. (b) Western blotting analyses for the effect of HDAC2 inhibitor VPA on CGRP-evoked iNOS, RIG-I, and NF- $\kappa$ B protein expression in microglial cells with treatment of CGRP and pretreatment with VPA. Relative amounts of proteins were calculated by normalizing to GAPDH. All values are expressed as the means \pm SEMs $\left(N=4\right.$ independent cell culture preparations, Mann-Whitney $U$ tests or Kruskal-Wallis tests). ${ }^{*} P<0.05$ versus controls; ${ }^{*} P<0.05$ versus CGRP only groups. 
almost all cultured microglial cells expressed CGRP receptor components CRLR, RAMP1, and CRCP. Treatment of microglial cells with CGRP also increased the HDAC2 protein level. These results suggested that CGRP operated via its receptor that might contribute to modulation of gene transcription through HDAC2-medated epigenetic mechanism [26]. In order to obtain insights into HDAC2 target gene function, GO and KEGG analysis annotations were applied to the HDAC2 target gene pool. GO terms for BP process categories showed that key higher genes related with positive regulation of $\mathrm{T}$ cell cytokine production, NOS biosynthetic process, and activation of NF- $\kappa \mathrm{B}$-inducing kinase activity. KEGG pathways for upregulated genes were mainly related to the Fc epsilon RI signaling pathway, RIG-like receptor signaling pathway, and NF- $\kappa \mathrm{B}$ signaling pathway. The expression of NOS, RIG-I, NF- $\kappa$ B was further verified by Western blot in microglia following CGRP treatment. Therefore, alterations of this histone deacetylase induced by CGRP could have severe consequences on microglial activation at the level of transcription [5].

HDAC2 has been shown to regulate microglial activation and induce cytokine expression through the NF- $\kappa \mathrm{B}$ pathway $[15,17]$. Previous data indicated that HDAC2 activates NF- $\kappa \mathrm{B}$ and promotes NF- $\kappa \mathrm{B}$-dependent gene expression [16], which plays a crucial role in microglial activation [17]. NF- $\kappa \mathrm{B}$ can interact with corepressors HDAC1 and HDAC2 to regulate gene transcription [27, 28]. Activation of NF- $\kappa$ B seems essential for the transcription of most of the proinflammatory molecules, such as cytokines and chemokines [29]. A previous report showed that activation of $\mathrm{NF}-\kappa \mathrm{B}$ regulates microglial conversion to a proinflammatory type [30]. In the present study, we found CGRP increased the expression of HDAC2 and $\mathrm{NF}-\kappa \mathrm{B}$ in microglia, suggesting that they might coordinately regulate the gene transcription for microglial activation induced by CGRP.

CGRP has been reported to activate microglia directly to produce proinflammatory mediators, including NOS and cytokines [31-33]. The specific recruitment of HDAC2 to $\mathrm{NF}-\kappa \mathrm{B}$ at target promoters and the consequent effects on acetylation status may play an important role in regulating iNOS as well as other NF- $\kappa \mathrm{B}$-dependent genes involved in inflammation [27, 34]. Activation of microglia displays NO production via iNOS activity which upregulates microglial phagocytosis and increases TRPV2 expression [35]. Our experiment showed that CGRP increased the expression of iNOS, NF- $\kappa$ B, and RIG-I protein levels in microglia after CGRP treatment. Nonetheless, these increases in iNOS, NF$\kappa \mathrm{B}$, and RIG-I expression were inhibited by HDAC2 inhibitor, suggesting that increases of iNOS, NF- $\kappa$ B, and RIG-I expression in microglia by CGRP might be associated with HDAC2. Furthermore, activation of RIG-I may induce the expression of proinflammatory cytokines through the activation of $\mathrm{NF}-\kappa \mathrm{B}[36,37]$. A previous study demonstrated that HDAC2 inhibitor VPA reduced induction of RIG-I expression in different cancer cell lines by decitabine [38], suggesting that HDAC2 might be involved in regulation of RIG-I expression. Taken together with our results, these indicate that the upregulation of HDAC2 by CGRP may contribute to immune and inflammatory responses through the NO/iNOS, NF- $\kappa \mathrm{B}$, and RIG-I signal pathways in microglial activation [39].

\section{Conclusions}

In summary, we systematically evaluated CGRP-mediated HDAC2 enrichments in microglial cells and gained new insights into links between key pathways and HDAC2 induced by CGRP in the microglial activation. Genomic analyses suggested that genes with the differential HDAC2 enrichments induced by CGRP function in diverse cellular pathways and many are involved in immune and inflammatory responses. However, further studies are needed to confirm our results.

\section{Data Availability}

The data used to support the findings of this study are available from the corresponding authors upon request.

\section{Conflicts of Interest}

None of the authors has a conflict of interest to declare.

\section{Authors' Contributions}

Xingjing Guo and Dan Chen contributed equally to this work.

\section{Acknowledgments}

ChIP-seq experiments were performed by KangChen Biotech, Shanghai, China. The study was supported by the National Natural Science Foundation of China (Nos. 31871215 and 81371234) and Natural Science Foundation of Shandong Province of China (ZR2019MH027).

\section{Supplementary Materials}

Table S1: differentially enriched regions for up and down peak-promoter-annotation. (Supplementary Materials)

\section{References}

[1] K. Li, Y.-H. Tan, A. R. Light, and K.-Y. Fu, "Different peripheral tissue injury induces differential phenotypic changes of spinal activated microglia," Clinical and Developmental Immunology, vol. 2013, Article ID 901420, 8 pages, 2013.

[2] T. L. Tay, N. Hagemeyer, and M. Prinz, "The force awakens: insights into the origin and formation of microglia," Current Opinion in Neurobiology, vol. 39, no. 1, pp. 30-37, 2016.

[3] J. T. Malon, S. Maddula, H. Bell, and L. Cao, "Involvement of calcitonin gene-related peptide and CCL2 production in CD40-mediated behavioral hypersensitivity in a model of neuropathic pain," Neuron Glia Biology, vol. 7, no. 2-4, pp. 117128, 2011.

[4] J. Priller, C. A. Haas, M. Reddington, and G. W. Kreutzberg, "Calcitonin gene-related peptide and ATP induce immediate early gene expression in cultured rat microglial cells," Glia, vol. 15, no. 4, pp. 447-457, 1995.

[5] M. Reddington, J. Priller, J. Treichel, C. Haas, and G. W. Kreutzberg, "Astrocytes and microglia as potential targets for 
calcitonin gene related peptide in the central nervous system," Canadian Journal of Physiology and Pharmacology, vol. 73, no. 7, pp. 1047-1049, 1995.

[6] L. Yi-Zhen, M. Run-Pei, Y. Tian-Yuan et al., "Mild mechanic stimulate on acupoints regulation of CGRP-positive cells and microglia morphology in spinal cord of sciatic nerve injured rats," Frontiers in Integrative Neuroscience, vol. 13, 2019.

[7] L.-J. Zhou, J. Peng, Y.-N. Xu et al., "Microglia are indispensable for synaptic plasticity in the spinal dorsal horn and chronic pain," Cell Reports, vol. 27, no. 13, pp. 38443859.e6, 2019.

[8] S. Iyengar, M. H. Ossipov, and K. W. Johnson, "The role of calcitonin gene-related peptide in peripheral and central pain mechanisms including migraine," Pain, vol. 158, no. 4, pp. 543-559, 2017.

[9] C. Sardi, L. Zambusi, A. Finardi et al., "Involvement of calcitonin gene-related peptide and receptor component protein in experimental autoimmune encephalomyelitis," Journal of Neuroimmunology, vol. 271, no. 1-2, pp. 18-29, 2014.

[10] J. Li, C. V. Vause, and P. L. Durham, "Calcitonin gene-related peptide stimulation of nitric oxide synthesis and release from trigeminal ganglion glial cells," Brain Research, vol. 1196, no. 1, pp. 22-32, 2008.

[11] Y. Kawarai, S. Orita, J. Nakamura et al., "Changes in proinflammatory cytokines, neuropeptides, and microglia in an animal model of monosodium iodoacetate-induced hip osteoarthritis," Journal of Orthopaedic Research, vol. 36, no. 11, pp. 2978-2986, 2018.

[12] R. J. Cady, J. R. Glenn, K. M. Smith, and P. L. Durham, “Calcitonin gene-related peptide promotes cellular changes in trigeminal neurons and glia implicated in peripheral and central sensitization," Molecular Pain, vol. 7, pp. 1744-8069, 2011.

[13] M. Datta, O. Staszewski, E. Raschi et al., "Histone deacetylases 1 and 2 regulate microglia function during development, homeostasis, and neurodegeneration in a context-dependent manner," Immunity, vol. 48, no. 3, pp. 514-529.e6, 2018.

[14] M. S. Sung, H. Heo, G. H. Eom et al., "HDAC2 regulates glial cell activation in ischemic mouse retina," International Journal of Molecular Science, vol. 20, no. 20, p. 5159, 2019.

[15] B. S. Durham, R. Grigg, and I. C. Wood, "Inhibition of histone deacetylase 1 or 2 reduces induced cytokine expression in microglia through a protein synthesis independent mechanism," Journal of Neurochemistry, vol. 143, no. 2, pp. 214224, 2017.

[16] T. Wagner, N. Kiweler, K. Wolff et al., "Sumoylation of HDAC2 promotes NF- $\kappa \mathrm{B}$-dependent gene expression," Oncotarget, vol. 6, no. 9, pp. 7123-7135, 2015.

[17] F. Z. Jiao, Y. Wang, H. Y. Zhang, W. B. Zhang, L. W. Wang, and Z. J. Gong, "Histone deacetylase 2 inhibitor CAY10683 alleviates lipopolysaccharide induced neuroinflammation through attenuating TLR4/NF- $\kappa \mathrm{B}$ signaling pathway," Neurochemical Research, vol. 43, no. 6, pp. 1161-1170, 2018.

[18] S. An, G. Zong, Z. Wang, J. Shi, H. du, and J. Hu, "Expression of inducible nitric oxide synthase in mast cells contributes to the regulation of inflammatory cytokines in irritable bowel syndrome with diarrhea," Neurogastroenterology and Motility, vol. 28, no. 7, pp. 1083-1093, 2016.

[19] T. I. Lee, S. E. Johnstone, and R. A. Young, "Chromatin immunoprecipitation and microarray-based analysis of protein location," Nature Protocols, vol. 1, no. 2, pp. 729-748, 2006.
[20] B. Langmead and S. L. Salzberg, "Fast gapped-read alignment with Bowtie 2," Nature Methods, vol. 9, no. 4, pp. 357-359, 2012.

[21] J. Feng, T. Liu, and Y. Zhang, "Using MACS to identify peaks from ChIP-Seq data," Current Protocols in Bioinformatics, vol. 34, no. 1, pp. 2.14.1-2.14.14, 2011.

[22] M. Haeussler, A. S. Zweig, C. Tyner et al., "The UCSC Genome Browser database: 2019 update," Nucleic Acids Research, vol. 47, no. D1, pp. D853-D858, 2019.

[23] M. Ashburner, C. A. Ball, J. A. Blake et al., "Gene ontology: tool for the unification of biology. The Gene Ontology Consortium," Nature Genetics, vol. 25, no. 1, pp. 25-29, 2000.

[24] M. Kanehisa, Y. Sato, M. Kawashima, M. Furumichi, and M. Tanabe, "KEGG as a reference resource for gene and protein annotation," Nucleic Acids Research, vol. 44, no. D1, pp. D457-D462, 2016.

[25] C. Wu, A. Li, J. Hu, and J. Kang, "Histone deacetylase 2 is essential for LPS-induced inflammatory responses in macrophages," Immunology \& Cell Biology, vol. 97, no. 1, pp. 7284, 2019.

[26] C. A. Warwick, L. P. Shutov, A. J. Shepherd, D. P. Mohapatra, and Y. M. Usachev, "Mechanisms underlying mechanical sensitization induced by complement C5a: the roles of macrophages, TRPV1, and calcitonin gene-related peptide receptors," Pain, vol. 160, no. 3, pp. 702-711, 2019.

[27] B. P. Ashburner, S. D. Westerheide, and A. S. Baldwin, "The p65 (RelA) subunit of NF-kappaB interacts with the histone deacetylase (HDAC) corepressors HDAC1 and HDAC2 to negatively regulate gene expression," Molecular and Cellular Biology, vol. 21, no. 20, pp. 7065-7077, 2001.

[28] Y. Chen, H. Wang, S. O. Yoon et al., "HDAC-mediated deacetylation of NF- $\kappa \mathrm{B}$ is critical for Schwann cell myelination," Nature Neuroscience, vol. 14, no. 4, pp. 437-441, 2011.

[29] O. T. Somade, B. O. Ajayi, O. A. Safiriyu, O. S. Oyabunmi, and A. J. Akamo, "Renal and testicular up-regulation of proinflammatory chemokines (RANTES and CCL2) and cytokines (TNF- $\alpha$, IL- $1 \beta$, IL-6) following acute edible camphor administration is through activation of NF-kB in rats," Toxicology Reports, vol. 6, no. 1, pp. 759-767, 2019.

[30] M. Galán-Ganga, Á. J. García-Yagüe, and I. Lastres-Becker, "Role of MSK1 in the induction of NF- $\kappa$ B by the chemokine CX3CL1 in microglial cells," Cellular and Molecular Neurobiology, vol. 39, no. 3, pp. 331-340, 2019.

[31] C. V. Vause and P. L. Durham, "CGRP stimulation of iNOS and NO release from trigeminal ganglion glial cells involves mitogen-activated protein kinase pathways," Journal of Neurochemistry, vol. 110, no. 3, pp. 811-821, 2009.

[32] F. R. Nieto, A. K. Clark, J. Grist, V. Chapman, and M. Malcangio, "Calcitonin gene-related peptide-expressing sensory neurons and spinal microglial reactivity contribute to pain states in collagen-induced arthritis," Arthritis \& Rheumatology, vol. 67, no. 6, pp. 1668-1677, 2015.

[33] R. Q. Sun, Y. J. Tu, N. B. Lawand, J. Y. Yan, Q. Lin, and W. D. Willis, "Calcitonin gene-related peptide receptor activation produces PKA- and PKC-dependent mechanical hyperalgesia and central sensitization," Journal of Neurophysiology, vol. 92, no. 5, pp. 2859-2866, 2004.

[34] Z. Yu, "Histone deacetylases augment cytokine induction of the iNOS gene," Journal of the American Society of Nephrology, vol. 13, no. 8, pp. 2009-2017, 2002. 
[35] M. J. E. Maksoud, V. Tellios, D. An, Y. Y. Xiang, and W. Y. $\mathrm{Lu}$, "Nitric oxide upregulates microglia phagocytosis and increases transient receptor potential vanilloid type 2 channel expression on the plasma membrane," Glia, vol. 67, no. 12, pp. 2294-2311, 2019.

[36] P. Lagana, L. Soraci, M. E. Gambuzza, G. Mancuso, and S. A. Delia, "Innate Immune Surveillance In The Central Nervous System Following Legionella Pneumophila Infection," CNS \& Neurological Disorders-Drug Targets, vol. 16, no. 10, pp. 1080-1089, 2018.

[37] M. Yu and S. J. Levine, "Toll-like receptor, RIG-I-like receptors and the NLRP3 inflammasome: key modulators of innate immune responses to double-stranded RNA viruses," Cytokine and Growth Factor Reviews, vol. 22, no. 2, pp. 63-72, 2011.

[38] D. Bensaid, T. Blondy, S. Deshayes et al., "Assessment of new HDAC inhibitors for immunotherapy of malignant pleural mesothelioma," Clinical Epigenetics, vol. 10, no. 1, 2018.

[39] L. Carniglia, D. Ramírez, D. Durand et al., "Neuropeptides and microglial activation in inflammation, pain, and neurodegenerative diseases," Mediators Of Inflammation, vol. 2017, Article ID 5048616, 23 pages, 2017. 